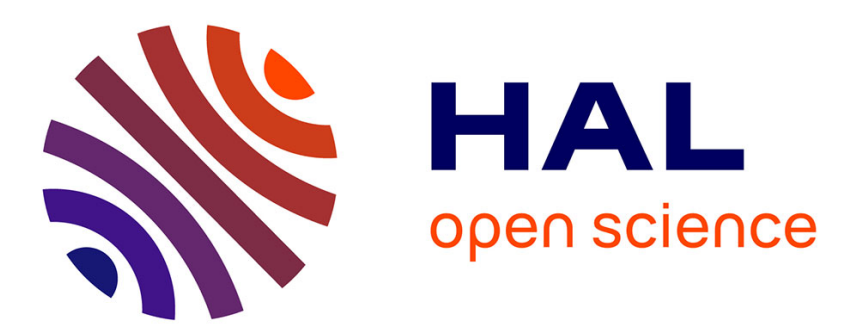

\title{
Weak Localization and Time Reversal of Ultrasound in a Rotational Flow
}

\author{
Julien de Rosny, Arnaud Tourin, Arnaud Derode, Philippe Roux, Mathias \\ Fink
}

\section{> To cite this version:}

Julien de Rosny, Arnaud Tourin, Arnaud Derode, Philippe Roux, Mathias Fink. Weak Localization and Time Reversal of Ultrasound in a Rotational Flow. Physical Review Letters, 2005, 95, pp.074301. 10.1103/PhysRevLett.95.074301 . hal-00018725

\section{HAL Id: hal-00018725 \\ https://hal.science/hal-00018725}

Submitted on 8 Feb 2006

HAL is a multi-disciplinary open access archive for the deposit and dissemination of scientific research documents, whether they are published or not. The documents may come from teaching and research institutions in France or abroad, or from public or private research centers.
L'archive ouverte pluridisciplinaire HAL, est destinée au dépôt et à la diffusion de documents scientifiques de niveau recherche, publiés ou non, émanant des établissements d'enseignement et de recherche français ou étrangers, des laboratoires publics ou privés. 


\title{
Weak localization and time reversal of ultrasound in a rotational flow
}

\author{
Julien de Rosny, ${ }^{*}$ Arnaud Tourin, Arnaud Derode, Philippe Roux, and Mathias Fink \\ Laboratoire Ondes et Acoustique, C.N.R.S. - UMR 7587, E.S.P.C.I., \\ Université Paris 7, 10 rue Vauquelin, 75231 Paris, France
}

(Dated: February 8, 2006)

\begin{abstract}
A one-channel time-reversal (TR) experiment is performed inside a rough reverberating cavity in the presence of a rotational flow. The amplitude of the TR wave is plotted versus the distance between the TR channel and the initial source: when they coincide, it exhibits a "time-reversal enhancement" (TRE). With no flow, the TRE is the same as the coherent backscattering enhancement (CBE). But contrary to CBE, the TRE peak is shown to be insensitive to the breaking down of reciprocity due to the flow. This new effect of weak localization is sustained by a diagrammatic approach.
\end{abstract}

PACS numbers: 43.20.+g, 43.35.+d, 73.20.Fz, 42.25.Dd

Many experiments have shown that coherent interferences of waves survive in random media despite disorder. These interferences play a crucial role in the well-known coherent backscattering enhancement (CBE). The CBE was first observed in optics. Experimentally, it appears as a peak in the angular distribution of the backscattered intensity (albedo): at exact backscattering, the mean intensity is twofold enhanced. The CBE is considered as a sign of weak localization of waves propagating in a disordered heterogeneous medium. Weak localization originates from the constructive interferences between a multiple scattering path and its reciprocal counterpart which follows exactly the same sequence of scatterers but in a reverse order. The main features of weakly localized waves are insensitive to many aspects of the random media such as dispersion or absorption. Indeed, as was first predicted by Golubentsev[1], only reciprocity breaking in the propagation medium can alter the constructive interferences and destroy the enhancement. Partial suppression of the CBE by Faraday rotation of light in a multiple scattering medium was the first experimental evidence of the disappearance of weak localization[2].

Here, we present a new experimental manifestation of weak localization. Just like the CBE, it manifests itself as an enhancement in a particular direction; but contrary to the CBE, it is insensitive to a break down of reciprocity! The experiment takes benefit of a time-reversal (TR) device for ultrasonic waves. A point-like source emits a short ultrasonic pulse in a multiple scattering medium. The backscattered wavefield is recorded by the TR device which is close to the source but can be moved around it. The recorded waveform is flipped and sent back. The time-reversed wave propagates in the same scattering medium, and a TR amplitude pattern is measured, i.e., we study the amplitude of the wave that is recreated at the initial source point with respect to the distance between this point and the TR device. The experiment is redone for different realizations of disorder: the mean TR amplitude pattern is found to be exactly the same as the intensity distribution due to the CBE.
Precisely, it shows up an enhancement by a factor of two when the source and the TR device coincide. We will refer to this phenomenon as the "time-reversal enhancement" (TRE). In a recent paper[3] we have discussed the theoretical distinction between TRE and CBE. In particular, it was shown that contrary to CBE, the background of the TRE pattern is due to weak localization while the peak itself simply results from incoherent interferences. In terms of the diagrammatic formalism of the multiple scattering theory, the TRE is due to ladder diagrams while the background results from the most-crossed diagrams, which is the exact opposite of what happens in a $\mathrm{CBE}$ experiment. However the experiments presented in ref. [3] were performed in a reciprocal medium, where these two contributions are the same. Therefore the intrinsic difference between $\mathrm{CBE}$ and TRE could not be revealed. In this Letter, we compare CBE and TRE in the case of a multiply scattered ultrasonic wave in the presence of a rotating water flow that breaks reciprocity. We show that the TRE remains unperturbed by the flow, while the $\mathrm{CBE}$ is destroyed because of the reciprocity breaking. It clearly appears that CBE and TRE are two different manifestations of weak localization.

The experimental setup is drawn in Fig. 1. A cylinder with four blades linked to an electric motor creates the rotating flow. Depending on the electrical potential applied to the motor, the speed of rotation of the cylinder goes from $0 \mathrm{~Hz}$ to $2.6 \mathrm{~Hz}$. The flow is confined by a motionless plexiglass cylinder. $5 \mathrm{~cm}$ below the blades is opened an aperture to insert a linear array made of 64 transducers whose central working frequency is $3.4 \mathrm{MHz}$. Each transducer is $10-\mathrm{mm}$ high and $0.417-\mathrm{mm}$ wide. Therefore the beam is horizontally confined. In order to increase the reflectivity of the boundaries, a 0.5 -mm thick stainless steel sheet covers the inner boundary of the plexiglass cylinder. About one hundred of stainless steel rods have been glued on the stainless sheet parallel to the cylinder axis in order to obtain a quasi-2D cavity with rough boundaries. After 2-3 diffusions off the boundaries (travel time of $200-300 \mu s$ ), the field becomes diffusive. Firstly the 

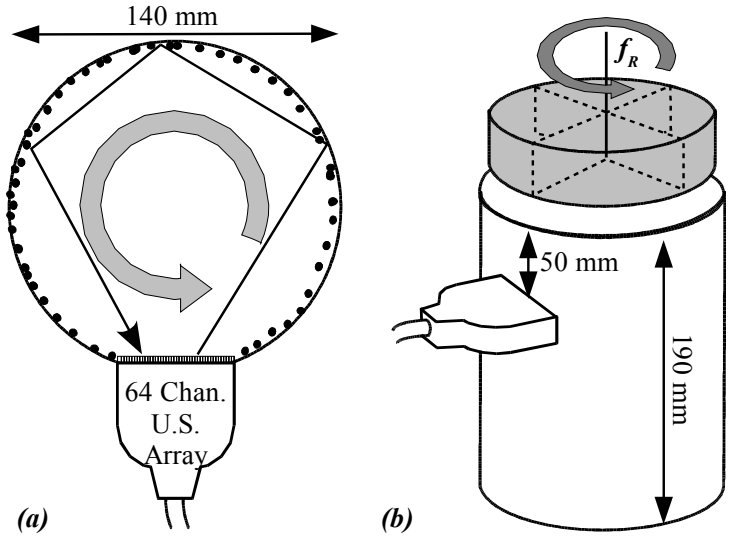

FIG. 1: Experimental setup: quarter view.

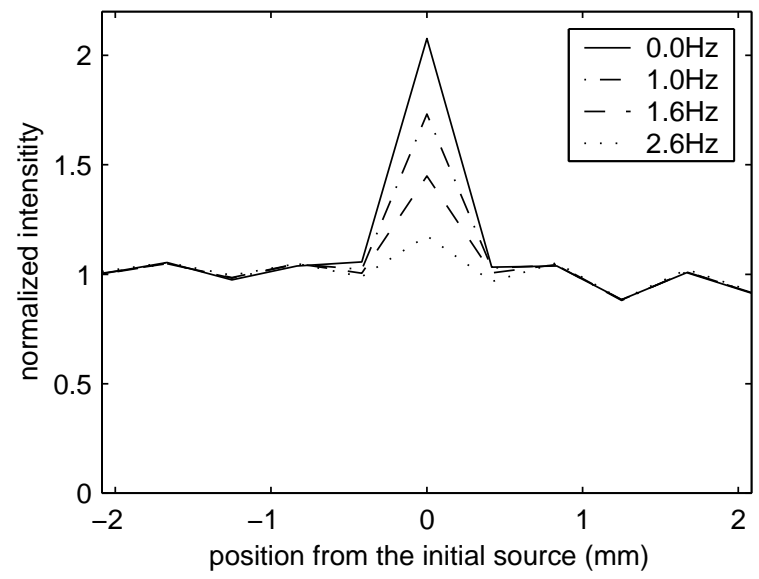

FIG. 2: Normalized distribution of the averaged backscattered intensity integrated between 500 and $800 \mu$ s after the pulse at different rotating frequencies of the motor. The normalization constant is the average intensity recorded at the outer elements (background) when the rotating frequency is $0 \mathrm{~Hz}$.

CBE is measured. To this end, a short pulse is emitted by one element of the array. Then the time dependence of the pressure field between 500 and $800 \mu$ s after the pulse emission is recorded on 11 elements centered on the emitting one. Within this time window, the wave has been reflected more than 5 times off the rough boundaries. The square of the pressure field is integrated at each transducer. These operations are averaged 54 times, the emitting element on the array changing each time. Assuming the cavity to be ergodic, a good estimate of the average intensity is obtained. In Fig. 2 is plotted the intensity distribution with respect to the relative distance from the initial source. A twofold enhancement clearly appears when there is no flow. Since CBE is recorded inside a cavity, the typical width of the peak is one wavelength (near field cone)[4]. Now as the rotation speed increases, the peak amplitude decreases continuously while

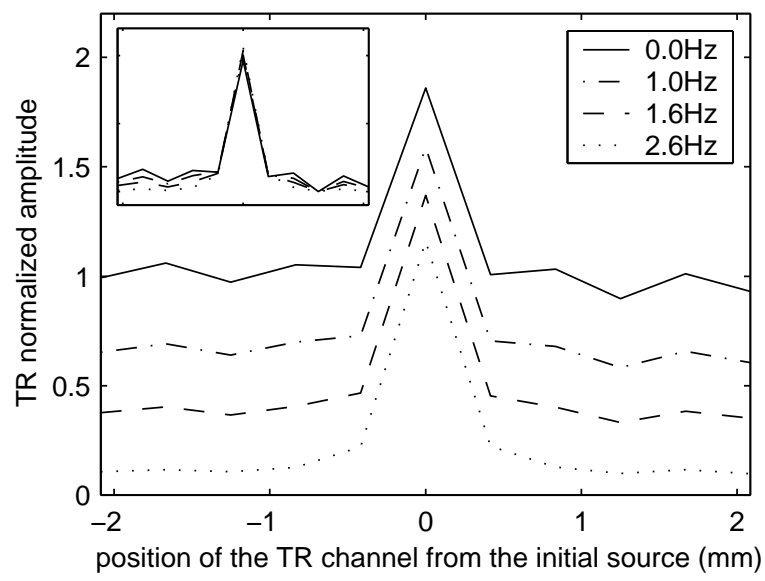

FIG. 3: The amplitude of the TR pulse recreated at the initial time and at the initial source array element with respect to the distance to the time reversal array element for different rotating frequencies of the motor. The normalization procedure is the same as the one used for CBE. In the inner subplot, the background level has been subtracted.

the background level does not change. Exactly the same behavior for CBE has been observed in optics where the reciprocity was broken by a magnetic field[2]. For the time reversal experiment, the procedure is a bit more complex. First, a short pulse is emitted by one transducer. The same or another transducer plays the role of the point-like TR device. It records the pressure field between $500 \mu \mathrm{s}$ and $800 \mu \mathrm{s}$ after the pulse emission. $1 \mathrm{~ms}$ later, the signal is sent back into the medium but in reverse order. Then the resulting field is recorded by the element that was first used as a source. The relative position of the TR element to the initial source one goes from $-2.08 \mathrm{~mm}$ to $2.08 \mathrm{~mm}$ (i.e., 11 transducers). During the TR step, the flow distribution must be the same as the one encountered during the initial emission step. To check this stationarity condition, $1 \mathrm{~ms}$ after the TR focusing step, the initial emission step is redone and the pressure response recorded on the TR channel is compared to the initial one. The two responses are identical. The repetition of this test over the entire flow speed range confirms that our experiment can be considered as stationary at least at the scale of the 2 steps of the TR process. In order to have a better estimate, the entire TR procedure is averaged on 54 different positions on the array. The quantity we measure is the amplitude of the wave that is recreated at the source with respect to the distance between the source and the TR element. Similar to the CBE when there is no flow, it shows up a peak, a TRE (see Fig. 3). However in presence of flow, the evolution is completely different: the more the vorticity increases, the more the background level decreases while the peak amplitude does not change!

In terms of harmonic Green's functions, the mean in- 
(a)
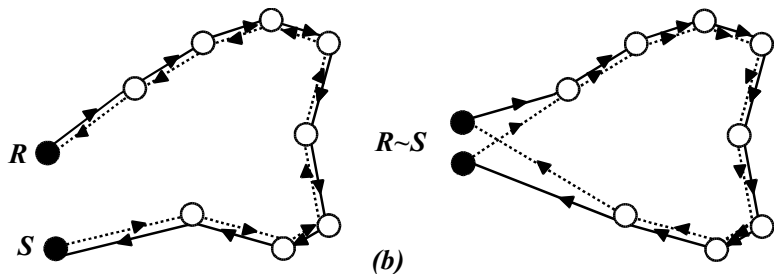

FIG. 4: Schematic representation of the two contributions to the TR focusing. The arrows represent the propagation direction and a dotted line represents a conjugate amplitude path. (a) is the main contribution to TR focusing. (b) is the enhanced contribution to TR.

tensity recorded at point $\mathbf{R}$ due to a source at $\mathbf{S}$, can be written

$$
I \propto\left\langle G(\mathbf{S}, \mathbf{R}, \Omega) G^{*}(\mathbf{S}, \mathbf{R}, \Omega)\right\rangle
$$

where $G(\mathbf{A}, \mathbf{B}, \Omega)$ is the Green's function between points $\mathbf{A}$ and $\mathbf{B}$ and $\Omega$ represents the flow vorticity distribution. The mean amplitude of the TR field recorded at the initial source location $\mathbf{S}$ after time reversal at $\mathbf{R}$ can be written

$$
\Psi_{R T} \propto\left\langle G(\mathbf{R}, \mathbf{S}, \Omega) G^{*}(\mathbf{S}, \mathbf{R}, \Omega)\right\rangle
$$

Indeed, in a first step, a short pulse is emitted between $\mathbf{S}$ and $\mathbf{R}$. This response is time reversed which corresponds to phase conjugate it at each frequency. This signal is then emitted at $\mathbf{R}$ and the TR field recorded at $\mathbf{S}$ (see ref. [3] for more details). The factors of proportionality (not shown here) in Eqs. (1) and (2) lead to the correct dimensions for the intensity $I$ and the amplitude $\Psi_{R T}$. Note that $I$ and $\Psi_{R T}$ are identical only when $\mathbf{S}=\mathbf{R}$ and in this case $\Psi_{R T}>0$ whatever $\Omega$. Indeed, it has been shown that $\mathrm{TR}$ in a random medium requires at least reciprocity. With a rotational flow, reciprocity no longer survives $[G(\mathbf{R}, \mathbf{S}, \Omega) \neq G(\mathbf{S}, \mathbf{R}, \Omega)]$ except when $\mathbf{R}=\mathbf{S}$ where it becomes "trivially" true. A schematic representation of the multiple scattering process involved in the TR is very helpful (Fig. 4). Two distinct contributions appear. The first one is always present. It weakly depends on the relative distance between $\mathbf{S}$ and $\mathbf{R}$. This contribution is sensitive to the reciprocity of the propagating medium because two conjugated paths in this contribution travel in opposite directions. When $\mathbf{R}$ is close enough to $\mathbf{S}$, a new contribution appears. Contrary to the previous one, this contribution does not depend on reciprocity. In order to go further, the diagrammatic expansions of the vertex implied in the CBE and the TR are performed in Fig. 5. The diagrammatic conventions of Rytov et al. [5] are employed here. In this diagrammatic representation, the propagation always goes from the left to the right. A thick horizontal bar represents a Green's function. When a vertical tick crosses a horizontal thick bar, the conjugated Green's function is considered. Two dots linked by a dashed line represent the

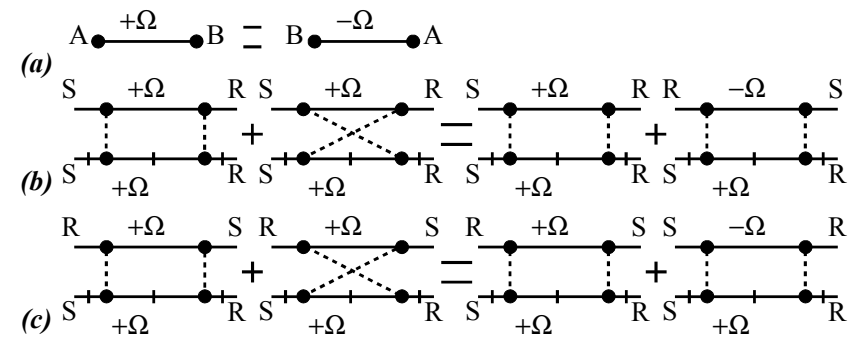

FIG. 5: Generalization of the reciprocity theorem (a). Double scattering diagrammatic representation of: (b) the mean intensity between point $\mathbf{S}$ and $\mathbf{R}$ and (c) one channel time reversal (phase conjugate). In Figs. (b) and (c), the first and the second diagrams on the left hand of the diagrammatic equation respectively correspond to the ladder and the most-crossed diagrams. At the right hand, the most-crossed diagrams have been rewritten using the energy conservation principle (cf. fig. (a)).

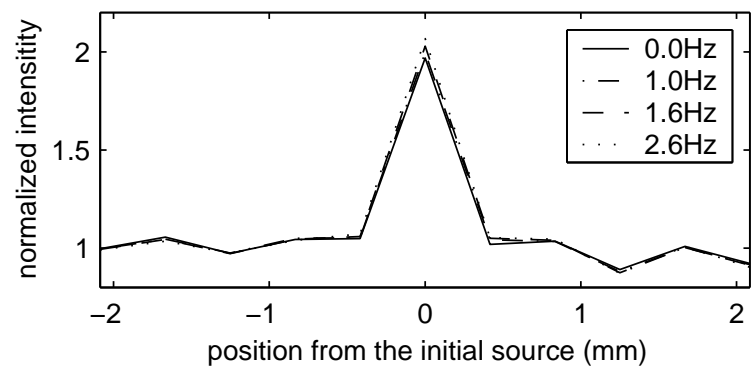

FIG. 6: This figure is obtained by summing the CBE and the TRE distributions for different rotation speeds (Fig. 2 and Fig. 3 are added)

same scatterer. It clearly appears that contrary to CBE, the most-crossed diagrams always contribute to the TRE whatever the positions of the source and TR channel are because a same position ( $\mathbf{S}$ or $\mathbf{R}$ ) is linked to a same scatterer. As for the ladder diagrams, they only contribute to the TR focusing when the source and the TR channel are at the same location. Therefore in this particular case, the TRE and CBE are the same. Finally, whatever the vorticity, when CBE and TRE are added the symmetry between the background and the enhancement contributions is restored and the factor 2 is recovered. This statement is confirmed experimentally in Fig. 6. In the multiple scattering theory, the contribution of the mostcrossed diagrams is computed by transforming it into a ladder-like contribution. To this end, the "reciprocity theorem" that exchanges the role of the source and the receiver has to be invoked. Due to time-reversal invariance, in a medium with a vorticity distribution $\Omega$, the response from $\mathbf{A}$ to $\mathbf{B}$ is equal to the response from $\mathbf{B}$ to $\mathbf{A}$, but with the opposite vorticity distribution $-\Omega$ [ $G(\mathbf{A}, \mathbf{B}, \Omega)=G(\mathbf{B}, \mathbf{A},-\Omega)]$. The diagrammatic representation of this property is shown in Fig. 5a. Inserting this relation in the most-crossed contributions leads to 

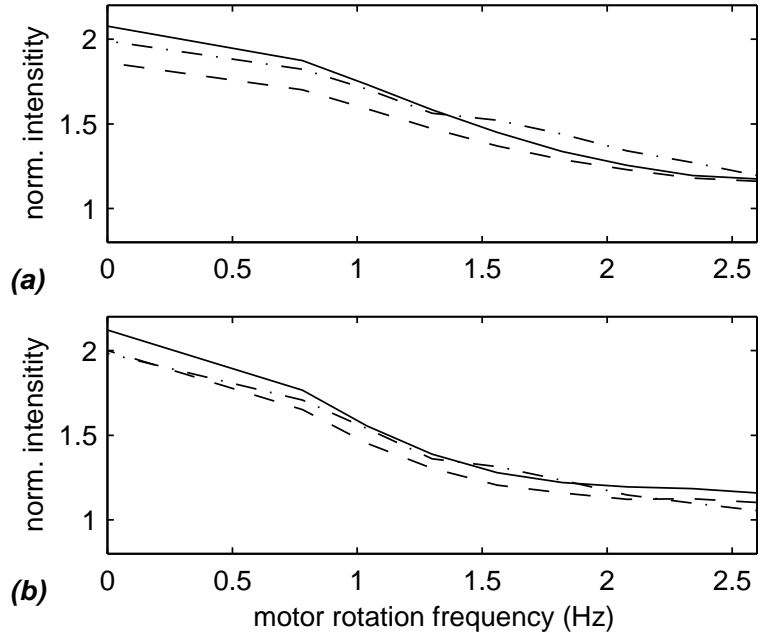

FIG. 7: Experimental peak amplitude of CBE (continuous line) and TRE (dashed line) with respect to the motor speed. Curves (a) and (b) are respectively obtained for a CBE and TRE time windows centered on $650 \mu$ s and $1150 \mu \mathrm{s}$. Both windows are $300-\mu$ s wide. The dashed-dotted curves correspond to the theoretical prediction.

right hand sides of diagrammatic equations in Figs. 5b and c. The opposite vorticities yield a dephasing $\Delta \phi$ between two conjugated paths that differs from 0 . Consequently one can show that the most-crossed contributions are attenuated by a factor $\langle\exp (i \Delta \phi)\rangle$ compared to the ladder ones[6]. The dephasing for a sequence of scatterers can be expressed as path integral

$$
\Delta \phi=2 \frac{k}{c} \oint \mathbf{v} d \mathbf{l}=2 \frac{k}{c} \int \Omega d A
$$

where $\mathbf{v}$ is the flow map, $k$ the wavenumber and $c$ the sound speed. Invoking Green's theorem, this expression can be written as a surface integral of the vorticity $(\Omega=\nabla \wedge \mathbf{v})$. To compute Eq. (3), one should have an idea of the structure of the flow. To this end, an ultrafast two-dimensional ultrasonic speckle velocimetry imaging technique has been used[7]. We observed that the flow rotates as a solid over a diameter of $80 \mathrm{~mm}$. In such a case the vorticity $\Omega$ is constant within this area. Therefore $\langle\exp (i \Delta \phi)\rangle$ only depends on the distribution of areas $A$. The acoustic paths going from and arriving at the source position define the area edges. Due to the large number of scattering, the distribution of $A$ is approximatively Gaussian. For simplicity two successive scatterings are assumed completely decorrelated. Then one can show that the variance of the surface $\left\langle A^{2}\right\rangle$ is $R^{3} c t\left\langle\sin (2 \theta)^{2}\right\rangle /\langle 2 \cos (\theta)\rangle$, where $\theta$ is the scattering angle that goes from $-\pi / 2$ to $\pi / 2$ and $R$ the radius. Assuming an isotropic scattering, it comes

$$
\left\langle e^{i \Delta \phi}\right\rangle \approx \exp \left(\frac{-\pi^{3} R^{3} \Omega^{2} t}{\lambda^{2} c}\right)
$$

In fig. 7 have been plotted the evolutions of the peak amplitude of $\mathrm{CBE}$ and TRE with respect to the rotating motor speed. It is confirmed that when $\mathbf{S}=\mathbf{R}$ the CBE and TRE amplitudes are the same. The vorticity $\Omega$ of the flow is experimentally obtained from the velocimetry survey. Eq. (4) with a radius $R$ set to $40 \mathrm{~mm}$ correctly fits the experimental results. $40 \mathrm{~mm}$ is lower than the $70 \mathrm{~mm}$ plexiglass cylinder radius. This difference has several origins. First of all, the real flow is not as simple as a pure solid rotation overall the cylinder. From the velocimetry measures, it appears that the flow is roughly solid over $80 \mathrm{~mm}$ diameter. Beyond, the flow speed decreases. Moreover some of the assumption may be not completely fullfilled. The surface distribution is not purely Gaussian and the scatterings are not completely isotropic and independent. Nevertheless, the theoretical results are qualitatively in agreement with the experimental ones.

Since the first observation of CBE with optical waves, it is known that stationary and dynamic CBE are powerful tools in order to measure the transport mean free path $\left(\ell^{*}\right)$ and the diffusion constant $D$. However CBE disappears as soon as reciprocity breaks down. Interestingly, in such situations the TRE could be used to evaluate $\ell^{*}$ or $D$. For instance, it could evaluate the effects of a strong magnetic field on these transport quantities associated to a multiply scattered optical wave.

The authors wish to thank Eric Larose and Bart Van Tiggelen for fruitful discussions as well as the research group IMCODE (groupe de recherches 2253, CNRS) for its financial support.

* Electronic address: julien.derosny@espci.fr; URL: http: //www.loa.espci.fr/ julien

[1] A. Golubentsev, Zh. Eksp. Teor. Fiz 86, 47 (1984), [Sov. Phys. JETP 59, 26 (1984)].

[2] F. Erbacher, R. Lenke, and G. Maret, Euro. Phys. Lett. 5, 551 (1993).

[3] J. de Rosny, A. Tourin, A. Derode, B. van Tiggelen, and M. Fink, Phys. Rev. E 70, 046601 (pages 12) (2004).

[4] J. de Rosny, A. Tourin, and M. Fink, Phys. Rev. Lett. 84, 1693 (2000); R. L. Weaver and O. I. Lobkis, Phys. Rev. Lett. 84, 4942 (2000).

[5] S. Rytov, I. Kravtsov, and V. Tatarskii, Principles of statistical radiophysics, Vol. 4, Wave propagation through random media (Springer-Verlag Berlin New York, 1989).

[6] E. Akkermans and G. Montanbaux, Physique mesoscopique des électrons et des photons (CNRS Edition EDP Sciences, 2004), chap. 6, pp. 215-287.

[7] L. Sandrin, S. Manneville, and M. Fink, Applied Physics Letters 78, 1155 (2001). 